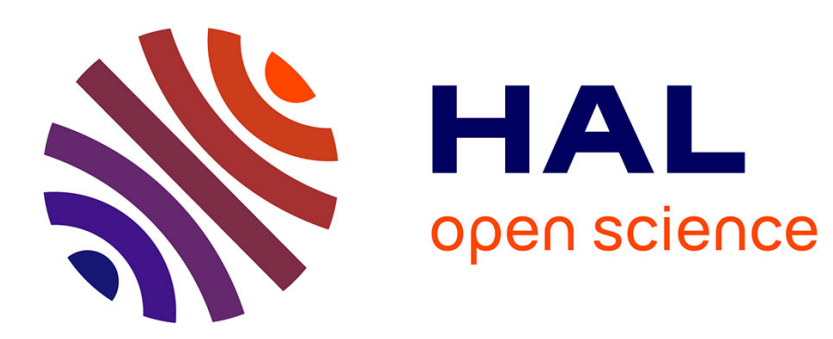

\title{
Shock-wave stability in quasi-mono-disperse granular materials
}

\author{
W.D. Neal, D.J. Chapman, W.G. Proud
}

\section{To cite this version:}

W.D. Neal, D.J. Chapman, W.G. Proud. Shock-wave stability in quasi-mono-disperse granular materials. European Physical Journal: Applied Physics, 2012, 57 (3), 10.1051/epjap/2011110054 . hal00788687

\section{HAL Id: hal-00788687 \\ https://hal.science/hal-00788687}

Submitted on 15 Feb 2013

HAL is a multi-disciplinary open access archive for the deposit and dissemination of scientific research documents, whether they are published or not. The documents may come from teaching and research institutions in France or abroad, or from public or private research centers.
L'archive ouverte pluridisciplinaire HAL, est destinée au dépôt et à la diffusion de documents scientifiques de niveau recherche, publiés ou non, émanant des établissements d'enseignement et de recherche français ou étrangers, des laboratoires publics ou privés. 


\title{
Shock-wave Stability in Quasi-mono-disperse Granular Materials
}

\author{
W.D. Neal, D.J. Chapman, and W.G. Proud ${ }^{a}$ \\ Institute of Shock Physics, Imperial College London, London, UK, SW7 2AZ
}

Received: date / Revised version: date

\begin{abstract}
This study investigated the possibility of sustaining steady shock-waves in brittle granular materials and thus determined the appropriateness of representing materials such as sand by the RankineHugoniot Jump Conditions. A series of plate impact experiments on quasi-mono-disperse glass microspheres were conducted with the aim of measuring shock velocity and identifying some typical features of shockwaves within granular materials. Granular bed thickness and input stress were varied to study the effects of dispersion on shock-wave velocity $\left(U_{s}\right)$. It has been shown that it is possible to sustain steady shock-waves in brittle granular materials due to no measurable wave dispersion occurring.
\end{abstract}

\section{Introduction}

Granular materials are vital to blast mitigating structures due to their dispersive nature and affordability. Research into the shock response of sand has typically measured Hugoniot relationships $\left(U_{s}=C_{0}+S u_{p}\right)$ using the Rankine-Hugoniot jump conditions (conservations of mass, momentum and energy) to infer densification.

It has been suggested that these relationships may not be appropriate to describe a brittle granular material [6] due to particle fracture and therefore potentially large lateral particle movement during shock compression. Lateral

Send offprint requests to: William Neal

a Present address: 4.80B, Royal School of Mines, Imperial College London, South Kensington, London, UK, SW7 2AZ particle movement would cause momentum to be transferred laterally and would not conserve it in the single dimension that the jump conditions dictate. Therefore the shock-wave would attenuate and be unsteady. There are three criteria for a steady shock-wave [1]:
1. $\frac{d a}{d P}>0$
2. $a+u_{p} \geq U_{s}$
3. $U_{s}>a_{0}$

For a shock-wave to be steady, and therefore make a Hugoniot relationship applicable, all three criteria must be satisfied. If the stability criteria are satisfied, the shockwave will steepen with time throughout the material. This happens until the effect of dissipational forces within the material become similar to the effect of increasing sound 
speed: the effects balance and the shock-wave becomes steady. If the effect of dissipational forces is larger than the effect of increasing sound speed the wave will become less steep and disperse. In this case the shock-wave will be unsteady.

This study aims to experimentally determine wether a granular material can support a steady shock-wave and therefore if the jump conditions should be used to produce Hugoniot relationships for granular materials.

\section{Methodology}

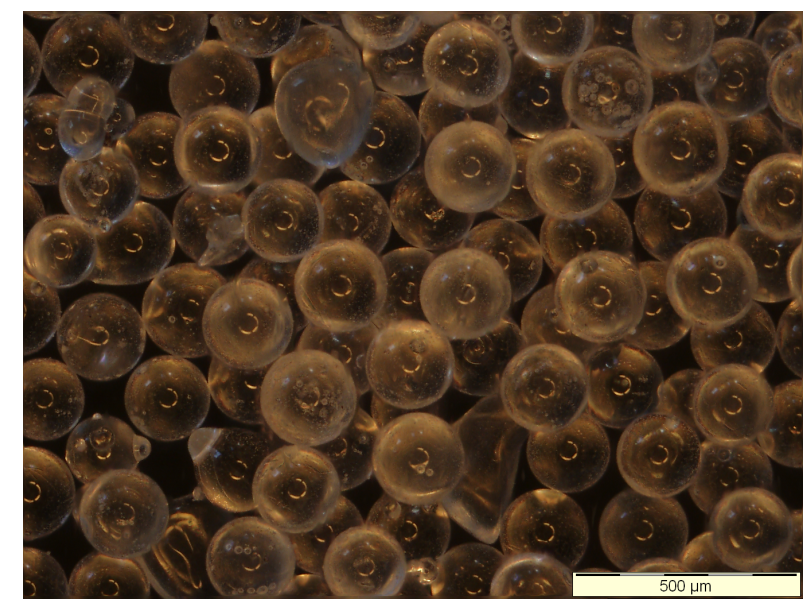

Fig. 1. Microscopy image of glass microspheres.

\subsection{Experiment}

Plate impact experiments were conducted on beds of glass microspheres of differing thicknesses to represent shock evolution over a granular bed.

\subsection{Granular Material}

Spherical soda-lime glass microspheres were chosen as a quasi-homogeneous granular material to improve the repeatability of the granular bed between experiments. Granular beds of sand are difficult to reproduce due to the complex shape and size distribution of the sand particles: . The particle size distribution was measured using a Mastersizer 2000 laser particle size analyser where the mean particle size was $194.9 \mu \mathrm{m}$. A sample of the microspheres was inspected using an optical microscope to verify particle sphericity (see figure 1). The particles were mostly spherical with few irregular shapes. The manufacturer stated the theoretical maximum density (TMD) to be $\rho_{0}=2.43-2.49 \mathrm{~g} \mathrm{~cm}^{-3}$.

The granular beds were contained by the cell design shown in figure 2. Two longitudinal stress gauges were sandwiched between PMMA plates before and aft of the granular bed. The gauges were bonded with epoxy resin. PMMA was selected to match the shock-impedance of the encapsulating plates to the epoxy resin. With differing impedances, the shock-wave would reverberate within the gauge adhesive and obscure features of the transmitted shockwave with additional wavelets. The plates were machined to \pm $4 \mu \mathrm{m}$ parallelism to ensure planar loading was achieved.

An aluminium ring confined the microspheres laterally. The longitudinal thickness of the rings was varied to enable stress to be measured after propagation through a range of bed thicknesses $(h=2,4$ and $6 \mathrm{~mm})$. These distances were measured at the plate centres $( \pm 4 \mu \mathrm{m})$ after construction to account for potential bending within the assembly (table 1). The internal diameter was measured to $\pm 0.02 \mathrm{~mm}$. 
Once assembled, the cells were filled with glass microspheres through a hole in the aluminium confining ring. During filling, the complete assembly was gently tapped 50 times with a small hammer at $\frac{1}{4}, \frac{1}{2}, \frac{3}{4}$ and full intervals to allow the particles to pack to a tapped density. The mass of microspheres in the cell was measured to \pm 0.01 $\mu \mathrm{g}$ and average density was calculated using measured dimensions to $\pm 0.04 \mathrm{~g} \mathrm{~cm}^{-3}$ (table 1$)$.

The encapsulation cells were subjected to planar impacts using a $50 \mathrm{~mm}$ light gas gun [4]. PMMA flyer plates were used to create symmetrical impacts for the lower stress shots and copper flyers were used for the higher stress ones. The surface of the front PMMA plate was aligned normal to the barrel and impact tilt was reduced to $\pm 2 \mathrm{mrad}$

\subsection{Diagnostics}

The velocity of the projectile was measured using arrays of graphite and brass shorting pins attached to the muzzle of the gun. The time of arrival of the flyer plate at four pairs of pins was measured and the impact velocity $\left(\mathrm{V}_{i}\right)$ was calculated to $\pm 1 \%$. (table 1 ) This velocity was used, in addition to shock-wave data for PMMA and copper [3], to calculate the shock velocity $\left(\mathrm{U}_{s}\right)$ within the front PMMA plate.

Piezo-resistive manganin stress gauges (LM-SS-125CH048) were used to measure the stress histories of the shock entering and leaving the granular bed. An oscilloscope (1 GS/s) recorded the voltage change across the gauge element. This temporal voltage history was converted to a

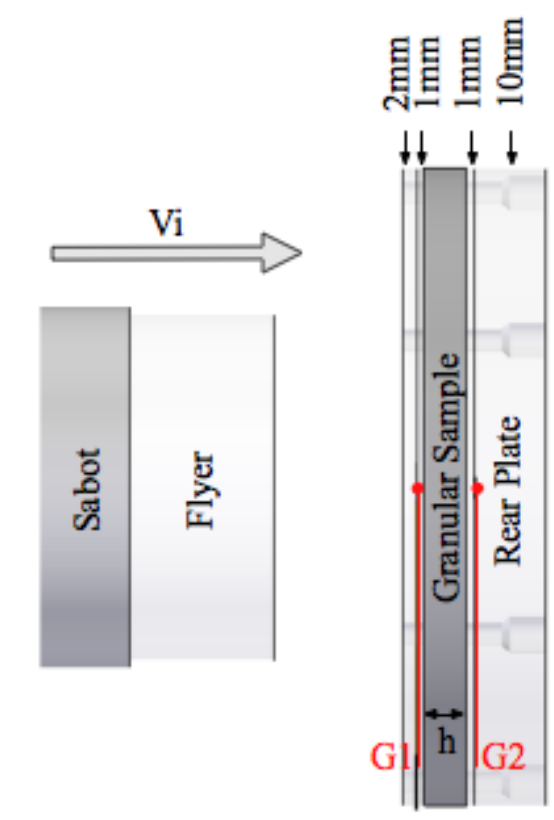

Fig. 2. Experimental design: projectile and encapsulating cell. longitudinal stress history using the method of Rosenberg et al.[2]

\section{Results and Discussion}

Table 1 summarises the plate impact experiments that were completed.

Figure 3 shows a typical calibrated stress history for a single experiment. All of the front gauge traces had shock rise times $<60 \mathrm{~ns}$ indicating a low impact tilt. A reflection is present in the front gauge trace due to the changing differences in shock impedance between the granular sample and the PMMA encapsulation. The initial partial release may be due to the very low impedance of the loose granular sample followed by a reflected shock from the higher impedance granular sample as it becomes rigid due to initial rearrangement of the microspheres. 
Table 1. Experiments completed.

\begin{tabular}{ccccc}
\hline$\sigma_{i}$ & $\mathbf{V}_{i}$ & Impactor & $h$ & $\rho_{00}$ \\
$(G P a)$ & $(\mathrm{mm} / \mu \mathrm{s})$ & & $(\mathrm{mm})$ & $\left(\mathrm{gcm}^{-1}\right)$ \\
\hline 3.31 & 0.793 & Copper & 4.001 & 1.59 \\
2.19 & 0.593 & Copper & 3.893 & 1.53 \\
1.53 & 0.791 & PMMA & 2.001 & 1.50 \\
1.55 & 0.784 & PMMA & 3.998 & 1.49 \\
1.05 & 0.587 & PMMA & 3.986 & 1.56 \\
1.08 & 0.592 & PMMA & 6.000 & 1.52 \\
0.66 & 0.391 & PMMA & 1.999 & 1.50 \\
0.66 & 0.389 & PMMA & 4.000 & 1.49 \\
0.66 & 0.389 & PMMA & 4.000 & 1.49 \\
0.65 & 0.388 & PMMA & 5.998 & 1.51 \\
0.48 & 0.289 & PMMA & 2.006 & 1.49 \\
0.46 & 0.284 & PMMA & 4.003 & 1.49 \\
\hline 0.37 & 0.230 & PMMA & 3.988 & 1.52 \\
\hline
\end{tabular}

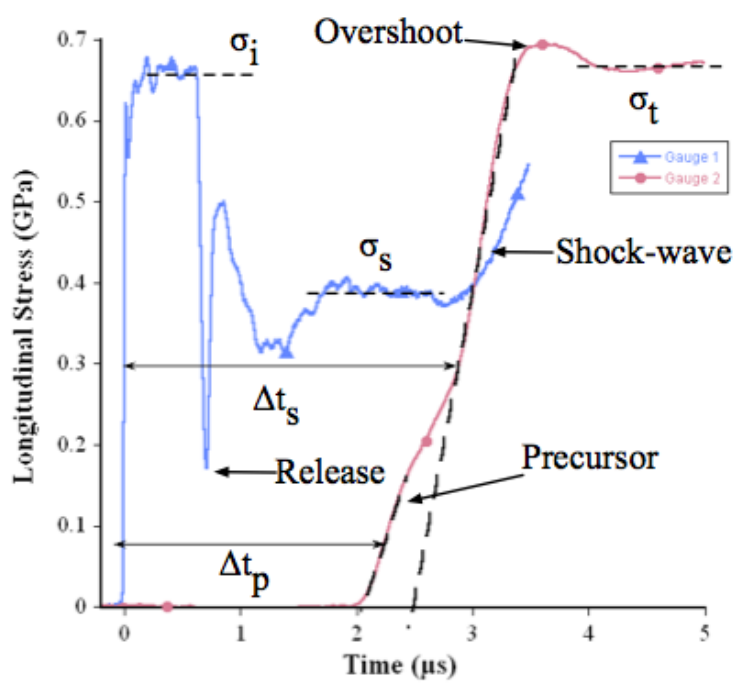

The stress level $\sigma_{s}$ has been identified in other studies of granular materials to be the stress in the PMMA relaxing to the Hugoniot stress in the sample [5] [7].

The transmitted stress profile shows a very ramped loading history. In the lower stress experiments, there are two distinct waves: a precursor wave and shock wave. This has been observed in other studies [8].

In all of the transmitted profiles there is evidence of an overshoot followed by a partial release to a constant plateau.

\subsection{Shock Evolution: Input Stress}

Figure 4 depicts changes in the transmitted stress profile compared to an increase in input stress. It is clear that the

Fig. 4. Transmitted shock-wave profiles. Profile labels indicate magnitude of input shock-wave $\left(\sigma_{i}\right)$.
Fig. 3. Typical stress data from a low $\sigma_{i}$ experiment. Blue and red lines indicate input and transmitted stress respectively. Time is relative to the shock arrival time in the front gauge.

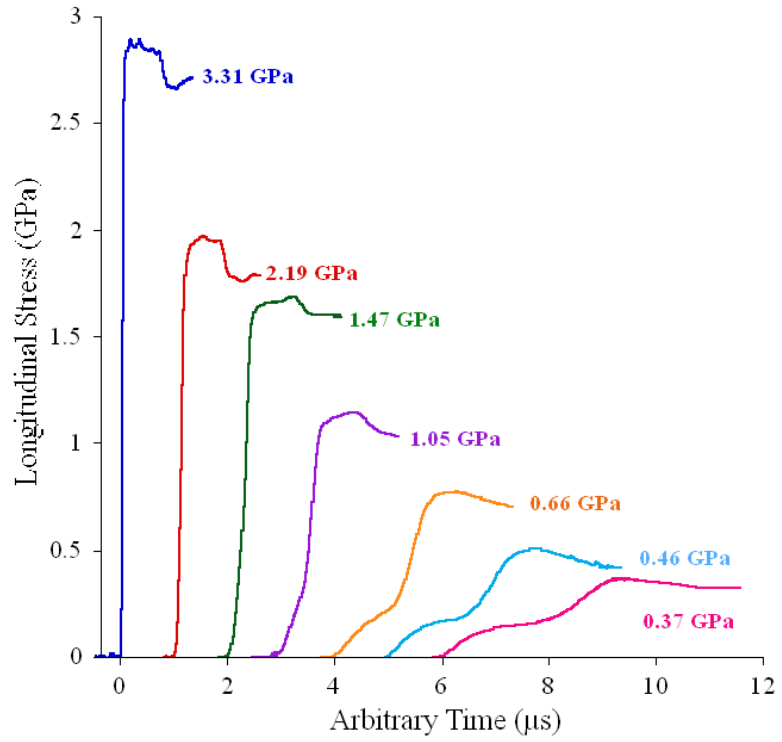




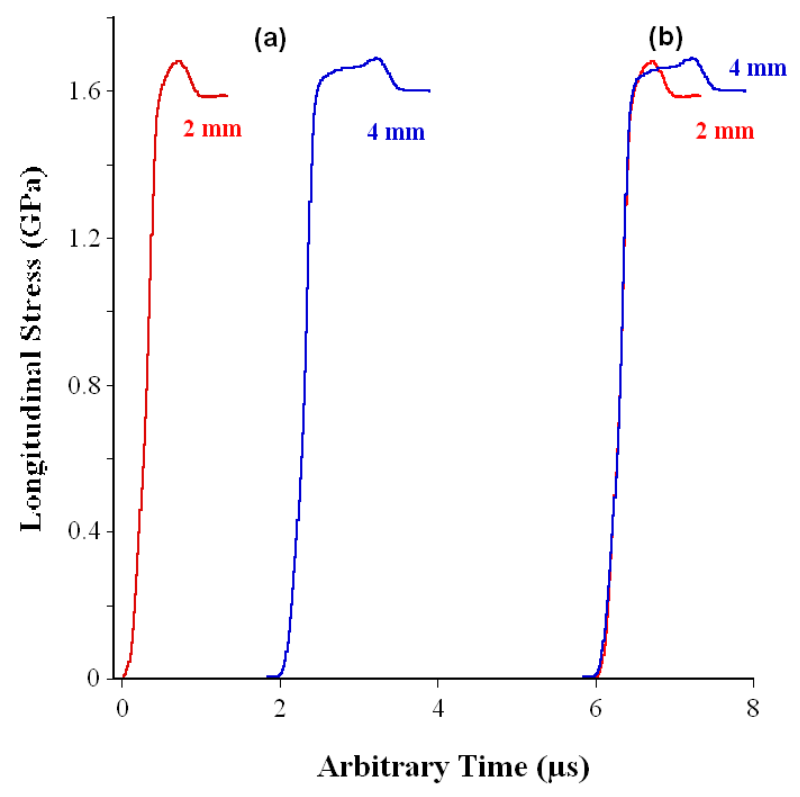

Fig. 5. Transmitted shock-wave profiles for $\sigma_{i}=1.47 \mathrm{GPa}$ (a) nominal time base and (b) normalised about the shock-wave time of arrival.

transmitted wave is very ramped at low input stresses. The precursor and shock-wave speeds generally increase with input stress as shown in figures 8 and 9 .

The overshoot feature of the transmitted wave has been observed in other studies on granular materials (sand) and was explained as relaxation due to particle fracture [8]. The traces in figure 4 show the magnitude of this overshoot stress to be proportional to input stress $\left(\sigma_{i}\right)$. If this theory is correct, the degree of particle fracture within the bed should also be proportional to $\sigma_{i}$. However, this is not conclusive and is part of ongoing investigations.

\subsection{Shock Evolution: Bed Thickness (h)}

\subsubsection{Over-Driven Wave Profiles}

Figure 5 shows the transmitted wave after $h=2$ and 4 $\mathrm{mm}$ of glass microspheres at $\sigma_{i}=1.47 \mathrm{GPa}$. There is no evidence of a precursor wave at this stress. The shockwave appears to be steady (b) as there is no change in gradient and no measurable attenuation ( $\sigma_{t}=$ constant $)$.

The duration of the overshoot feature of the transmitted pulse at $\sigma_{i}=1.47 \mathrm{GPa}$ is clearly proportional to the thickness of the granular bed $(h)$. If the amount of particles has increased within the bed then the amount of fracture would also increase thus increasing the fracture time and supporting the overshoot/fracture theory. This theory requires further investigation.

\subsubsection{Under-Driven Wave Profiles}

Figure 6 shows the transmitted wave after $h=2,4$ and $6 \mathrm{~mm}$ of glass microspheres at $\sigma_{i}=0.66 \mathrm{GPa}$. The precursor portion of the wave is clearly visible and does not appear to move at constant velocity (b). The gradient of the precursor wave continues to decrease with $h$ in the experiments where $\sigma_{i}=0.46 \mathrm{GPa}$ (figure 7 ). The measured velocity supports this observation (figure 9) which is determined by a linear fit of the wave profile. This is exaggerated by the decrease in gradient of the wave as $h$ increases but still dictates that the precursor wave is dispersing and is therefore unsteady.

When normalised, the gradient of the shock-wave portion of the transmitted wave does not appear to change 


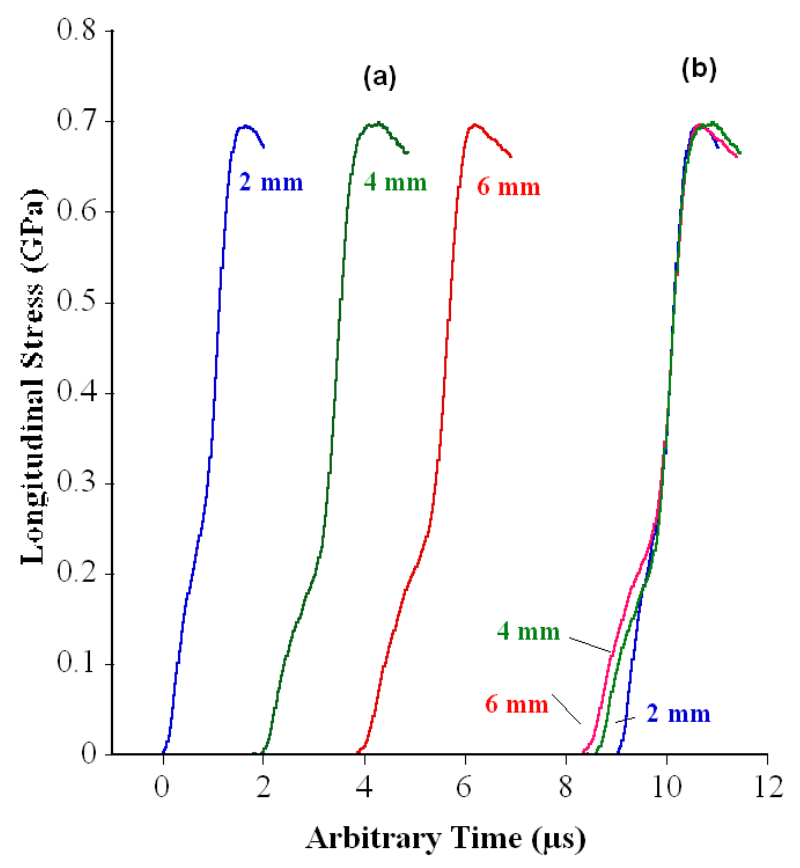

Fig. 6. Transmitted shock-wave profiles for $\sigma_{i}=0.66 \mathrm{GPa}$ (a) nominal time base and (b) normalised about the shock-wave.

with $h$ in any of the experiments. Therefore the shock stability criterion has been satisfied and the shock is steady.

\subsection{Wave Velocity}

Wave velocities were calculated by measuring the wave transit time between the two gauge positions. The mid point of the input shock and the mid points of linear fits of the transmitted wave profiles were used (see figure 3). The transit time through the PMMA plates was calculated using the Hugoniot for PMMA and subtracted from the overall transit time to give a sample wave transit time:

$$
U_{s}=\frac{h}{\Delta t_{s}-\Delta t_{P M M A}}
$$

It is clear from figure 8 that there is no measurable change in the $\mathrm{U}_{s}$ with $h$. This satisfies the shock stability criterion. The difference between $\mathrm{U}_{s}$ in the 1.05 and 1.47

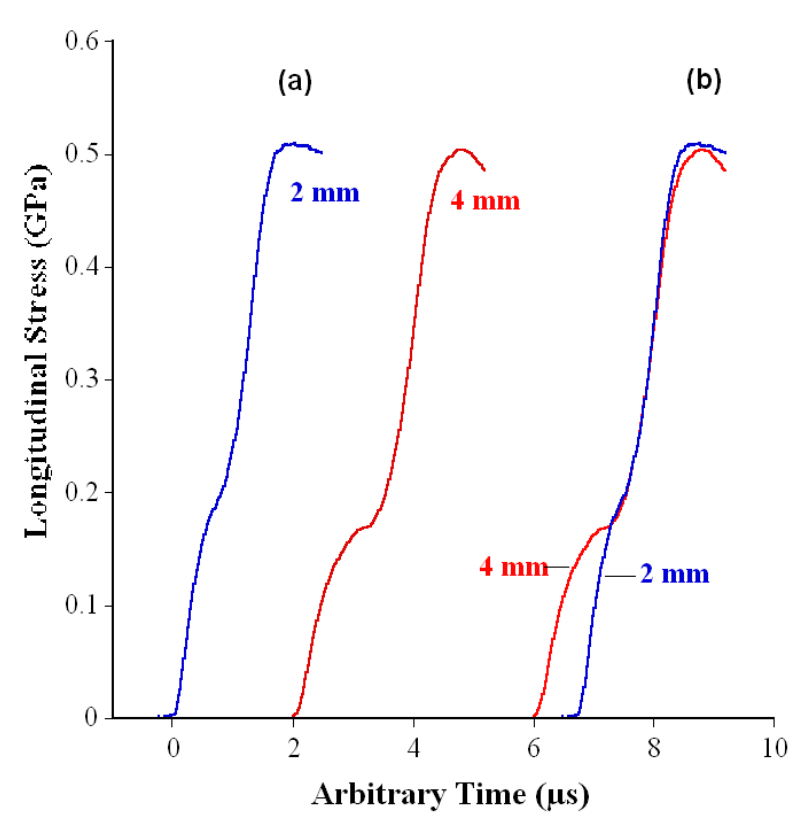

Fig. 7. Transmitted shock-wave profiles for $\sigma_{i}=0.46 \mathrm{GPa}$ (a) nominal time base and (b) normalised about the shock-wave.

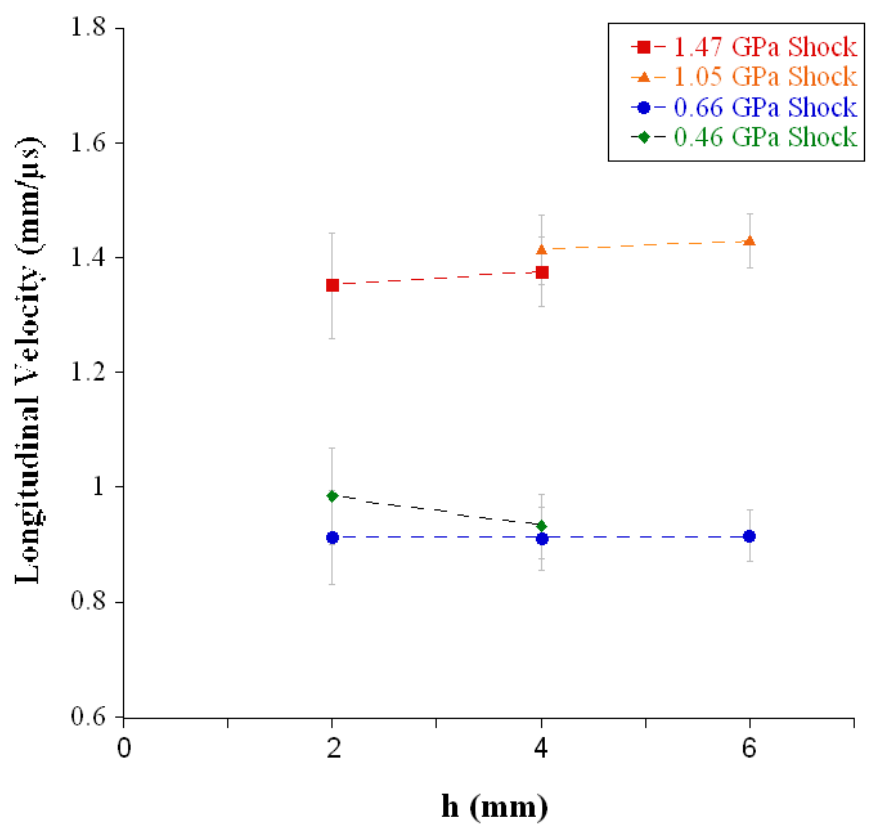

Fig. 8. The relationship between shock-wave velocity $\left(\mathrm{U}_{s}\right)$ and $h$. 


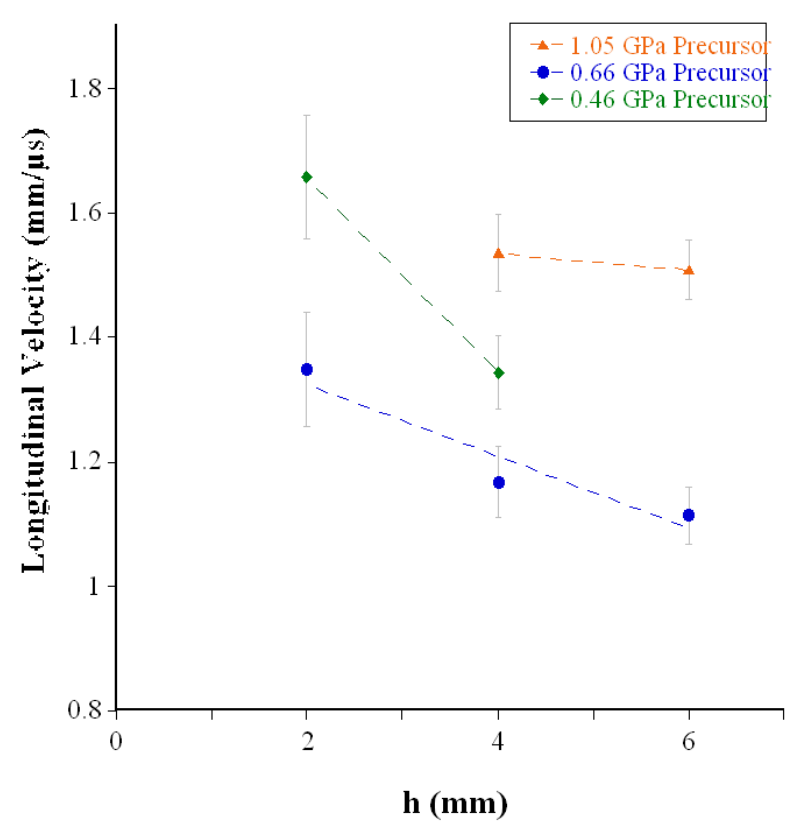

Fig. 9. The relationship between precursor wave velocity $\left(\mathrm{U}_{p r}\right)$ and $h$.

GPa experiments is very small. This small difference is consistent throughout all values of $h$ so it is unlikely to be erroneous.

It is clear that $\mathrm{U}_{p r}$ decreases with $h$ (figure 9) and that the wave disperses (figures 6 and 7 ). This could be explained by the force chains in the sample: a network of force carrying contacts in the granular system. In the $h$ $=2 \mathrm{~mm}$ bed, few particles form short force chains $(>10$ grains) and differences in time of arrival along those chains will be small. In the $4 \mathrm{~mm}$ and $6 \mathrm{~mm}$ samples, the force chains are longer ( $>20$ and $>30$ grains) and the differences in TOAs between force chains of differing lengths will be larger. As a linear increase in bed thickness occurs, a non linear increase in force chain length will occur and hence an apparent decrease in longitudinal precursor wave velocity. However, the gauge element represents a continuum response behind the centre of the sample bed and no single force chain can be observed. Proving this theory conclusively is limited by the investigators lack of spatially resolved diagnostics.

\section{Conclusions}

Plate impact experiments were conducted on quasi-monodisperse glass microspheres. Input stress $\left(\sigma_{i}\right)$ and bed thickness $(h)$ were varied to validate shock stability criteria and determine typical wave features. The following conclusions have been reached:

1. The shock-wave portion of the wave profiles satisfy the shock-wave stability criteria. Therefore the Hugoniot relationships can be applied to a brittle granular material.

2. The precursor wave $\left(\mathrm{U}_{p r}\right)$ does not appear to be steady and there is evidence of dispersion.

3. The overshoot feature of the transmitted pulse is proportional to bed thickness and input stress $\left(\sigma_{i}\right)$. This appears to agree with particle fracture theories [8] and therefore indicates that the degree of particle fracture may also be proportional to $\sigma_{i}$. However, further investigation is required to prove this theory.

\section{Acknowledgements}

The author would like to thank The Cavendish Laboratory for use of their gas gun and other facilities. In addition, the author is grateful for the assistance provided by the ISP Technician Team and AWE plc for funding. 


\section{References}

1. J.R. Asay, M. Shahinpoor, High-Pressure Shock Compression of Solids, (Springer-Verlag, 1993), pages 19-20.

2. Rosenberg et al., Journal of Applied Physics vol. 51 (1980) pp 3702

3. S. Marsh, LASL Shock Hugoniot Data, 1980

4. N.K. Bourne et al., Measurement Science and Technology vol. 6, num. 10 (1995), pp 1462

5. D. Chapman et al., Shock Compression of Condensed Matter vol.955 (2007), pp 955

6. D. Chapman et al., Proceedings of the 2006 SEM Annual Conference vol 2, pp 79

7. D. Chapman and K. Tsembelis. The behaviour of dry sand under shock-loading. AIP Conference ..., Jan 2006.

8. K. Tsembelis et al., DYMAT: Behaviour of Materials at High Strain Rates: Numerical Modelling 2002, p7963 